\title{
MITTEILUNGEN
}

\section{Repräsentation im Wahlkreis - Bevölkerung und Abgeordnete in Deutschland und Frankreich. Eine internationale wissenschaftliche Konferenz der DVParl am 7./8. November 2012 in Berlin}

Theorie und Empirie des Parlamentarismus stehen im Zentrum des Wirkens der Deutschen Vereinigung für Parlamentsfragen (DVParl). Zur Vermittlung einer schwierigen, stets entwicklungs- und erklärungsbedürftigen Regierungsform werden insbesondere Legitimationsund Repräsentationsfragen auf ihren Veranstaltungen behandelt, so auch am 7./8. November 2012 auf der internationalen Konferenz „Repräsentation im Wahlkreis - Bevölkerung und Abgeordnete in Deutschland und Frankreich“. Unter Leitung der DVParl-Vorsitzenden, MdB Brigitte Zypries, diskutierten zahlreiche französische und deutsche Abgeordnete und Wissenschaftler im Bundestag genau diese Fragen: Auf welche Weise erfüllen Abgeordnete in ihren Wahlkreisen die Aufgabe, für eine lebendige Verbindung zwischen Parlament und Bürgern zu sorgen? Wie erfahren sie von den Interessen, Meinungen und Wünschen ihrer Wähler? Wie informieren sie über politische Entscheidungen, geben Begründungen und schaffen Verständnis auch für unpopuläre Maßnahmen? Welche Rollen nehmen sie bei der Wahlkreisarbeit ein? Gleichsam in die andere Richtung wurde gefragt, wie die Bevölkerung die Parlamentarier bei ihrer Arbeit wahrnimmt, welche Wünsche und Ansprüche Bürger an Abgeordnete stellen und wie zufrieden sie mit ihren Leistungen sind.

Grundlage der Konferenz bildeten die ersten Ergebnisse des länderübergreifenden Forschungsprojekts CITREP („Citizens and Representatives in France and Germany“), das von der Deutschen Forschungsgemeinschaft (DFG) und ihrem französischen Pendant, der Agence Nationale de la Recherche (ANR), gefördert und an der Universität Stuttgart, der Martin-Luther-Universität Halle-Wittenberg und der Sciences Po Bordeaux durchgeführt wird. Seit 2010 untersuchen Wissenschaftler unter Leitung von Oscar W. Gabriel, Suzanne S. Schüttemeyer und Eric Kerrouche vergleichend den deutschen und französischen Alltag politischer Repräsentation im Wahlkreis. Neben den Einstellungen, Orientierungen und dem Verhalten der Abgeordneten bei der Wahrnehmung der Repräsentationsfunktion stehen die Einstellungen der Bürger gegenüber Abgeordneten, ihren Tätigkeiten und Leistungen im Mittelpunkt der Betrachtung. Für die Datenerhebung wurden jeweils ungefähr 60 Abgeordnete des Bundestages und der Assemblée Nationale mehrtägig begleitet, beobachtet und interviewt sowie repräsentative Bevölkerungsumfragen in beiden Ländern durchgeführt. Damit wird ein bislang kaum komparatistisch analysiertes Kernelement parlamentarischer Demokratie umfassend beleuchtet. ${ }^{1}$

Während der zweitägigen Tagung wurde in neun Panels ein faszinierender Einblick in die Situation der Abgeordneten vor Ort gegeben. Gleich zu Beginn wies Oscar W. Gabriel darauf hin, dass die Qualität der Repräsentationsbeziehung entscheidend für den Zustand der repräsentativen Demokratie ist. Im deutsch-französischen Vergleich falle die ähnliche Struktur

1 Zum Inhalt, ersten Ergebnissen, Publikationen und Medienberichten vgl. die Homepage des Forschungsprojektes, http://www.citrep.eu (Abruf am 10. März 2013). 
der Einstellungen auf, während es Unterschiede im Niveau der Ansprüche und der Zufriedenheit gebe. Französische Bürger hätten höhere Erwartungen gegenüber ihren Abgeordneten als deutsche, dafür jedoch niedrigere Zufriedenheitswerte. Deutsche äußerten, ein eher ambivalentes Verhältnis zu ihren Repräsentanten zu haben, während Franzosen wesentlich skeptischer gegenüber ihren gewählten Vertretern seien. Gabriels Fazit gibt Anlass zur Sorge: In beiden Ländern sind die Repräsentationsbeziehungen in keinem guten Zustand. In Deutschland sei sie „gestört“ und in Frankreich „massiv unter Druck“. Hinzu kommt, dass fast Zweidrittel der Befragten in den beiden Ländern eine klare Präferenz für eine direkte Ausgestaltung von Demokratie haben.

Dennoch wenden sich die Bürger, so die Vizepräsidentin des Bundestages Petra Pau, in allen Lebenslagen an ihre Volksvertreter: „Wir sind für alles zuständig. (...) In meinem Wahlkreis gibt es kein Thema, das nicht an mich heran getragen wird." Die hohe Erwartungshaltung führt unweigerlich zu „systematischen Enttäuschungen“, wie MdB Andrea Voßhoff zu berichten hatte: „Viele konkrete Probleme der Bürger (...) können von uns doch gar nicht gelöst werden“, da lokale Anliegen häufig nicht von der Bundesebene zu bearbeiten seien.

Den kritischen Wahrnehmungen und hohen Erwartungen der Bürger steht die stete Professionalisierung und enorme Arbeitsleistung der nationalen Volksvertreter gegenüber. Olivier Costa und Sven T. Siefken illustrierten beispielsweise, dass vier von fünf Mitgliedern des Bundestages mehr als zehn Stunden pro Tag in Wahlkreiswochen arbeiten, drei von fünf gar mehr als zehn, wobei fast 60 Prozent jedes Wochenende im Dienst sind. Dieses hohe Engagement ist deutliches Indiz für die Relevanz des Wahlkreises, die mithilfe von vier Funktionen analytisch fassbar gemacht werden kann: Hier werden die Abgeordneten (1) von ihren Parteien nominiert und stellen sich (2) dem Votum der Wählerschaft. Der Wahlkreis dient (3) als Informationsquelle für die parlamentarische Arbeit und ist (4) ein Forum, auf dem der Abgeordnete politisch kommunizieren kann. Im deutsch-französischen Vergleich sei eine unterschiedliche Akzentsetzung auszumachen, die wesentlich auf die jeweilige institutionelle Ausgestaltung der Repräsentationssysteme mit ihren unterschiedlichen Handlungsanreizen zurückzuführen sei. Bundestagsabgeordnete konzentrierten sich stärker auf Parteiarbeit, während ihre französischen Kollegen größeren Wert auf Bürgerkontakt legten und eine Ämterhäufung (Cumul des Mandats) anstrebten. Deutsche Parlamentarier nutzten den Wahlkreis viel stärker zur Informationsaufnahme für die eigene parlamentarische Arbeit als die Mitglieder der Assemblée Nationale, zum Beispiel bei Besuchen in Unternehmen und öffentlichen Einrichtungen. Besonders einprägsam illustrierte diese deutsche Eigenheit MdB Hans-Peter Bartels, der von der „Angst“ Gerhard Schröders vor den aus ihren Wahlkreisen nach sitzungsfreien Wochen kommenden Parlamentariern sprach: „Dann kamen die Abgeordneten alle wieder, angefüllt mit den Erlebnissen der letzten zwei Wochen [im Wahlkreis], was man ihnen so gesagt hat. Und das bringen sie auch wirklich mit, das wird dann auch wirklich besprochen."

Doch nicht nur die Arbeit unterscheidet sich im internationalen Vergleich. Die CITREPForscher präsentierten auch eine Typologie der Wahlkreisstile einzelner Abgeordneter, die sich entlang der Dimensionen Generalist/Spezialist und Zuhörer/politischer Führer entfaltet. Generell dominieren in Deutschland diejenigen, die zuhören und Generalisten sind. Dabei handelt es sich vor allem um die so genannten Zuhörer, Parteigänger und Dienstleister. Hierauf gab der Bundestagsabgeordnete Ernst Dieter Rossmann in der Diskussion allerdings zu bedenken, dass der Stil auch von den konkreten Umständen abhängig sei: „Während der Agenda 2010 waren alle SPD-Abgeordnete nur ,Leader 'in ihrem Wahlkreis.“ 
In Deutschland konnte diese zeitweise Rollendominanz sicherlich durch die enge Verzahnung zwischen Wahlkreis- und Parteiarbeit erreicht werden. Wie Abgeordnete mit ihren lokalen Parteiorganisationen interagieren, stellten Danny Schindler, Elisa Deiss-Helbig und Laure Squarcioni dar. Während für Bundestagsabgeordnete die Partei eine fortwährend große Bedeutung hat, ist sie für die Mitglieder der Assemblée Nationale nur von der Nominierung bis zur Wahl wichtig. Im Wahlkreisalltag spielt sie dann eine eher untergeordnete Rolle. Dagegen lässt sich in Deutschland eine hohe Anzahl an parteibezogenen Wahlkreisveranstaltungen identifizieren. Die Mitglieder des Bundestages agieren besonders häufig in der Rolle des Parteivertreters. Bemerkenswert dabei ist, dass sich der Parteienwettbewerb in diesem Rollenmuster nur moderat in der Wahlkreisarbeit niederschlägt.

In der Gesamtschau ist dem Lob des früheren französischen Parlamentspräsidenten Bernard Accoyer beizupflichten: Die Veröffentlichung der Ergebnisse kommt zum 50. Jahrestag der Unterzeichnung des Élysée-Vertrages zwischen Deutschland und Frankreich zum richtigen Zeitpunkt. Darüber hinaus ist zu unterstreichen, dass Abgeordnete mit ihrer Wahlkreisarbeit entscheidend dazu beitragen, die parlamentarische Repräsentationsfunktion zu erfüllen. Das Wissen um die Bedingungen und tatsächliche Ausgestaltung jener wichtigen und bisher nicht ausreichend beachteten Abgeordnetentätigkeit, das die CITREP-Forscher in Berlin zur Diskussion stellten, kann künftig jenen dienen, die sich ebenso wie die DVParl der Vermittlung der Wirkungsweisen parlamentarischer Demokratie verschrieben haben. Es kann dazu beitragen, dem bis zur Politikverdrossenheit ausufernden schlechten Ansehen von Parteien, Parlamenten und der Politik im Allgemeinen durch beständige Aufklärung entgegenzuwirken.

Alexander Kühne und Marcus Wittig 